\title{
A New Refinement of Generalized Hölder's Inequality and Its Application
}

\author{
Jingfeng Tian \\ College of Science and Technology, North China Electric Power University, Baoding, Hebei 071051, China \\ Correspondence should be addressed to Jingfeng Tian; tianjfhxm_ncepu@163.com
}

Received 24 May 2013; Accepted 2 September 2013

Academic Editor: Huy Qui Bui

Copyright (C) 2013 Jingfeng Tian. This is an open access article distributed under the Creative Commons Attribution License, which permits unrestricted use, distribution, and reproduction in any medium, provided the original work is properly cited.

We present a new refinement of generalized Hölder's inequality due to Vasić and Pečarić. Moreover, the obtained result is used to improve Beckenbach-type inequality due to Wang.

\section{Introduction}

If $a_{k} \geq 0, b_{k} \geq 0(k=1,2, \ldots, n), p>1$, and $(1 / p)+(1 / q)=$ 1 , then

$$
\sum_{k=1}^{n} a_{k} b_{k} \leq\left(\sum_{k=1}^{n} a_{k}^{p}\right)^{1 / p}\left(\sum_{k=1}^{n} b_{k}^{q}\right)^{1 / q} .
$$

The sign of inequality is reversed for $p<1, p \neq 0$ (for $p<0$; we assume that $\left.a_{k}, b_{k}>0\right)$. Inequality (1) and its reversed version are called Hölder's inequalities and are important in the study of inequalities and in the field of applied mathematics. The important inequalities have attracted interest of many mathematicians and have been improved as well as generalized in several different directions. For example, Barza et al. [1] presented matriceal versions of Hölder's inequality. Nikolova and Varošanec [2] obtained some new refinements of the classical Hölder's inequality by using a convex function. Tian and $\mathrm{Hu}$ [3] established a new reversed version of a generalized sharp Hölder's inequality. For more detailed expositions, the interested reader may consult [1-13] and the references therein. Among various generalizations of (1), Vasić and Pečarić in [14] presented the following interesting theorem.
Theorem A. Let $A_{i j} \geq 0(i=1,2, \ldots, n, j=1,2, \ldots, m)$.

(a) If $\beta_{j}$ are positive numbers, such that $\sum_{j=1}^{m}\left(1 / \beta_{j}\right) \geq 1$, then

$$
\sum_{i=1}^{n} \prod_{j=1}^{m} A_{i j} \leq \prod_{j=1}^{m}\left(\sum_{i=1}^{n} A_{i j}^{\beta_{j}}\right)^{1 / \beta_{j}} .
$$

(b) If $\beta_{1}>0, \beta_{j}<0(j=2,3, \ldots, m)$ and if $\sum_{j=1}^{m}\left(1 / \beta_{j}\right) \leq$ 1 , then

$$
\sum_{i=1}^{n} \prod_{j=1}^{m} A_{i j} \geq \prod_{j=1}^{m}\left(\sum_{i=1}^{n} A_{i j}^{\beta_{j}}\right)^{1 / \beta_{j}} .
$$

(c) If $\beta_{j}<0(j=1,2, \ldots, m)$, then

$$
\sum_{i=1}^{n} \prod_{j=1}^{m} A_{i j} \geq \prod_{j=1}^{m}\left(\sum_{i=1}^{n} A_{i j}^{\beta_{j}}\right)^{1 / \beta_{j}} .
$$

The main objective of this paper is to build some new refinements of inequalities (2), (3), and (4). Moreover, the obtained results will be applied to improve Beckenbach-type inequality which is due to Wang [15]. 


\section{A New Refinement of Generalized Hölder's Inequality}

In this section, we first prove the following lemma, which plays a crucial role in proving our main results.

Lemma 1. Let $X_{i j}>0$ and let $1-\sum_{j=1}^{m} X_{i j}^{\beta_{j}}>0(i=1,2, \ldots, n$, $j=1,2, \ldots, m)$.

(a) If $\beta_{j}>0(j=1,2, \ldots, m)$ and if $\sum_{j=1}^{m}\left(1 / \beta_{j}\right) \geq 1$, then

$$
\begin{gathered}
\prod_{j=1}^{m}\left(1-\sum_{i=1}^{n} X_{i j}^{\beta_{j}}\right)^{1 / \beta_{j}}+\sum_{i=1}^{n} \prod_{j=1}^{m} X_{i j} \\
\leq\left[1-\left(\sum_{i=1}^{n} X_{i 2}^{\beta_{2}}-\sum_{i=1}^{n} X_{i 1}^{\beta_{1}}\right)^{2}\right]^{1 / \beta^{*}},
\end{gathered}
$$

where $\beta^{*}=\max \left\{\beta_{1}, \beta_{2}\right\}$.

(b) If $\beta_{1}>0, \beta_{j}>0(j=2,3, \ldots, m)$ and if $\sum_{j=1}^{m}\left(1 / \beta_{j}\right) \leq$ 1 , then

$$
\begin{gathered}
\prod_{j=1}^{m}\left(1-\sum_{i=1}^{n} X_{i j}^{\beta_{j}}\right)^{1 / \beta_{j}}+\sum_{i=1}^{n} \prod_{j=1}^{m} X_{i j} \\
\geq\left[1-\left(\sum_{i=1}^{n} X_{i 2}^{\beta_{2}}-\sum_{i=1}^{n} X_{i 1}^{\beta_{1}}\right)^{2}\right]^{1 / \beta_{2}} .
\end{gathered}
$$

(c) If $\beta_{j}<0(j=1,2, \ldots, m)$, then

$$
\begin{gathered}
\prod_{j=1}^{m}\left(1-\sum_{i=1}^{n} X_{i j}^{\beta_{j}}\right)^{1 / \beta_{j}}+\sum_{i=1}^{n} \prod_{j=1}^{m} X_{i j} \\
\geq\left[1-\left(\sum_{i=1}^{n} X_{i 2}^{\beta_{2}}-\sum_{i=1}^{n} X_{i 1}^{\beta_{1}}\right)^{2}\right]^{1 / \beta_{2}} .
\end{gathered}
$$

Proof. (a) Without loss of generality, we assume that $\beta_{1} \leq \beta_{2}$.

Case 1 (when $0<\beta_{1}<\beta_{2}$ ). It implies that $1 / \beta_{2}>0$ and $\left(1 / \beta_{1}\right)-\left(1 / \beta_{2}\right)>0$. According to $\left(1 / \beta_{2}\right)+\left(1 / \beta_{2}\right)+\left(\left(1 / \beta_{1}\right)-\right.$ $\left.\left(1 / \beta_{2}\right)\right)+\left(1 / \beta_{3}\right)+\cdots+\left(1 / \beta_{m}\right) \geq 1$, by using inequality $(2)$, we have

$$
\begin{aligned}
{\left[1-\left(\sum_{i=1}^{n} X_{i 2}^{\beta_{2}}-\sum_{i=1}^{n} X_{i 1}^{\beta_{1}}\right)^{2}\right]^{1 / \beta_{2}} } \\
=\left[\left(1-\sum_{i=1}^{n} X_{i 1}^{\beta_{1}}\right)+\sum_{i=1}^{n} X_{i 2}^{\beta_{2}}\right]^{1 / \beta_{2}} \\
\times\left[\left(1-\sum_{i=1}^{n} X_{i 2}^{\beta_{2}}\right)+\sum_{i=1}^{n} X_{i 1}^{\beta_{1}}\right]^{1 / \beta_{2}} \\
\times\left[\left(1-\sum_{i=1}^{n} X_{i 1}^{\beta_{1}}\right)+\sum_{i=1}^{n} X_{i 1}^{\beta_{1}}\right]^{\left(1 / \beta_{1}\right)-\left(1 / \beta_{2}\right)} \\
\times \prod_{j=3}^{m}\left[\left(1-\sum_{i=1}^{n} X_{i j}^{\beta_{j}}\right)+\sum_{i=1}^{n} X_{i j}^{\beta_{j}}\right]^{1 / \beta_{j}}
\end{aligned}
$$

$$
\begin{aligned}
& \geq\left(1-\sum_{i=1}^{n} X_{i 1}^{\beta_{1}}\right)^{1 / \beta_{2}}\left(1-\sum_{i=1}^{n} X_{i 2}^{\beta_{2}}\right)^{1 / \beta_{2}} \\
& \quad \times\left(1-\sum_{i=1}^{n} X_{i 1}^{\beta_{1}}\right)^{\left(1 / \beta_{1}\right)-\left(1 / \beta_{2}\right)} \times \prod_{j=3}^{m}\left(1-\sum_{i=1}^{n} X_{i 1}^{\beta_{1}}\right)^{1 / \beta_{j}} \\
& +\sum_{i=1}^{n}\left[\left(X_{i 2}^{\beta_{2}}\right)^{1 / \beta_{2}}\left(X_{i 1}^{\beta_{1}}\right)^{1 / \beta_{2}}\right. \\
& \left.\left.=\prod_{j=3}^{m}\left(1-\sum_{i=1}^{n} X_{i j}^{\beta_{j}}\right)^{1 / \beta_{j}}+\sum_{i=1}^{n} \prod_{j=1}^{m} X_{i j}^{\beta_{1}}\right)^{\left(1 / \beta_{1}\right)-\left(1 / \beta_{2}\right)} \prod_{j=3}^{m}\left(X_{i j}^{\beta_{j}}\right)^{1 / \beta_{j}}\right]
\end{aligned}
$$

which means that the desired inequality (5) holds for $0<\beta_{1}<$ $\beta_{2}$.

Case 2 (when $\beta_{1}=\beta_{2}>0$ ). By applying inequality (2), we obtain

$$
\begin{aligned}
{\left[1-\left(\sum_{i=1}^{n} X_{i 2}^{\beta_{2}}-\sum_{i=1}^{n} X_{i 1}^{\beta_{1}}\right)^{2}\right]^{1 / \beta_{2}} } \\
=\left[\left(1-\sum_{i=1}^{n} X_{i 1}^{\beta_{1}}\right)+\sum_{i=1}^{n} X_{i 2}^{\beta_{2}}\right]^{1 / \beta_{2}} \\
\times\left[\left(1-\sum_{i=1}^{n} X_{i 2}^{\beta_{2}}\right)+\sum_{i=1}^{n} X_{i 1}^{\beta_{1}}\right]^{1 / \beta_{2}} \\
\times\left(1-\sum_{j=3}^{m}\left[\left(1-\sum_{i=1}^{n} X_{i j}^{\beta_{j}}\right)^{\beta_{1}}\right)^{1 / \beta_{2}}\left(1-\sum_{i=1}^{n} X_{i j}^{\beta_{j}}\right]^{1 / \beta_{j}} X_{i=1}^{\beta_{2}}\right)^{1 / \beta_{2}} \\
\quad \times \prod_{j=3}^{m}\left(1-\sum_{i=1}^{n} X_{i j}^{\beta_{j}}\right)^{1 / \beta_{j}} \\
+\sum_{j=1}^{m}\left(1-\sum_{i=1}^{n}\left[\left(X_{i 2}^{\beta_{2}}\right)^{1 / \beta_{2}}\left(X_{i j}^{\beta_{1}}\right)^{1 / \beta_{2}} \times \prod_{j=3}^{m}\left(X_{i j}^{\beta_{j}}\right)^{1 / \beta_{j}}\right]\right.
\end{aligned}
$$

That is, inequality (5) is true for $\beta_{1}=\beta_{2}>0$.

(b) If $\beta_{1}>0, \beta_{j}<0(j=2,3, \ldots, m)$, then $\left(1 / \beta_{1}\right)-$ $\left(1 / \beta_{2}\right)>0,\left(1 / \beta_{j}\right)<0(j=2,3, \ldots, m)$. By the same method as in Case 1 , we obtain the desired inequality (6).

(c) The proof of inequality (7) is similar to the one of inequality (5), and we omit it.

The proof of Lemma 1 is completed. 
Next, we present new refinements of inequalities (2), (3), and (4).

Theorem 2. Let $A_{i j} \geq 0(i=1,2, \ldots, n, j=1,2, \ldots, m)$, and let $s$ be any given natural number $(1 \leq s \leq n)$.

(a) If $\beta_{j}>0(j=1,2, \ldots, m)$ and if $\sum_{j=1}^{m}\left(1 / \beta_{j}\right) \geq 1$, then

$$
\begin{aligned}
\sum_{i=1}^{n} \prod_{j=1}^{m} A_{i j} \leq & {\left[\prod_{j=1}^{m}\left(\sum_{i=1}^{n} A_{i j}^{\beta_{j}}\right)^{1 / \beta_{j}}\right] } \\
& \times\left[1-\left(\frac{A_{s 2}^{\beta_{2}}}{\sum_{i=1}^{n} A_{i 2}^{\beta_{2}}}-\frac{A_{s 1}^{\beta_{1}}}{\sum_{i=1}^{n} A_{i 1}^{\beta_{1}}}\right)^{2}\right]^{1 / \beta^{*}},
\end{aligned}
$$

where $\beta^{*}=\max \left\{\beta_{1}, \beta_{2}\right\}$.

(b) If $\beta_{1}>0, \beta_{j}<0(j=2,3, \ldots, m)$ and if $\sum_{j=1}^{m}\left(1 / \beta_{j}\right) \leq$ 1 , then

$$
\begin{aligned}
\sum_{i=1}^{n} \prod_{j=1}^{m} A_{i j} \geq & {\left[\prod_{j=1}^{m}\left(\sum_{i=1}^{n} A_{i j}^{\beta_{j}}\right)^{1 / \beta_{j}}\right] } \\
& \times\left[1-\left(\frac{A_{s 2}^{\beta_{2}}}{\sum_{i=1}^{n} A_{i 2}^{\beta_{2}}}-\frac{A_{s 1}^{\beta_{1}}}{\sum_{i=1}^{n} A_{i 1}^{\beta_{1}}}\right)^{2}\right]^{1 / \beta_{2}} .
\end{aligned}
$$

(c) If $\beta_{j}<0(j=1,2, \ldots, m)$, then

$$
\begin{aligned}
\sum_{i=1}^{n} \prod_{j=1}^{m} A_{i j} \geq & {\left[\prod_{j=1}^{m}\left(\sum_{i=1}^{n} A_{i j}^{\beta_{j}}\right)^{1 / \beta_{j}}\right] } \\
& \times\left[1-\left(\frac{A_{s 2}^{\beta_{2}}}{\sum_{i=1}^{n} A_{i 2}^{\beta_{2}}}-\frac{A_{s 1}^{\beta_{1}}}{\sum_{i=1}^{n} A_{i 1}^{\beta_{1}}}\right)^{2}\right]^{1 / \beta_{2}} .
\end{aligned}
$$

Proof. Consider the following substitution:

$$
X_{i j}=\frac{A_{i j}}{\left(\sum_{k=1}^{n} A_{k j}^{\beta_{j}}\right)^{1 / \beta_{j}}} \quad(i=1,2, \ldots, n, j=1,2, \ldots, m) .
$$

It is easy to see that, for any given natural number $s(1 \leq$ $s \leq n)$, the following inequalities hold:

$$
X_{i j}>0, \quad 1-\sum_{1 \leq i \leq n, i \neq s} X_{i j}^{\beta_{j}}>0 .
$$

Consequently, by using the substitution (13) and inequality (5), we have

$$
\begin{aligned}
\prod_{j=1}^{m}[1 & \left.-\sum_{1 \leq i \leq n, i \neq s}\left(\frac{A_{i j}^{\beta_{j}}}{\sum_{k=1}^{n} A_{k j}^{\beta_{j}}}\right)\right]^{1 / \beta_{j}} \\
& +\sum_{1 \leq i \leq n, i \neq s}\left(\prod_{j=1}^{m} \frac{A_{i j}}{\left(\sum_{k=1}^{n} A_{k j}^{\beta_{j}}\right)^{1 / \beta_{j}}}\right)
\end{aligned}
$$

$$
\begin{aligned}
\leq\{1- & {\left[\sum_{1 \leq i \leq n, i \neq s}\left(\frac{A_{i 2}^{\beta_{2}}}{\sum_{k=1}^{n} A_{k 2}^{\beta_{2}}}\right)\right.} \\
& \left.\left.-\sum_{1 \leq i \leq n, i \neq s}\left(\frac{A_{i 1}^{\beta_{1}}}{\sum_{k=1}^{n} A_{k 2}^{\beta_{2}}}\right)\right]^{2}\right\}^{1 / \beta^{*}}
\end{aligned}
$$

for $\beta_{j}>0(j=1,2, \ldots, m), \sum_{j=1}^{m}\left(1 / \beta_{j}\right) \geq 1$, and thus we have

$$
\begin{gathered}
\frac{\prod_{j=1}^{m} A_{s j}}{\prod_{j=1}^{m}\left(\sum_{k=1}^{n} A_{k j}^{\beta_{j}}\right)^{1 / \beta_{j}}}+\frac{\sum_{1 \leq i \leq n, i \neq s} \prod_{j=1}^{m} A_{i j}}{\prod_{j=1}^{m}\left(\sum_{k=1}^{n} A_{k j}^{\beta_{j}}\right)^{1 / \beta_{j}}} \\
\leq\left[1-\left(\frac{A_{s 2}^{\beta_{2}}}{\sum_{k=1}^{n} A_{k 2}^{\beta_{2}}}-\frac{A_{s 1}^{\beta_{1}}}{\sum_{k=1}^{n} A_{k 1}^{\beta_{1}}}\right)^{2}\right]^{1 / \beta^{*}},
\end{gathered}
$$

that is,

$$
\begin{aligned}
& \frac{\sum_{i=1}^{n}\left(\prod_{j=1}^{m} A_{i j}\right)}{\prod_{j=1}^{m}\left(\sum_{k=1}^{n} A_{k j}^{\beta_{j}}\right)^{1 / \beta_{j}}} \\
& \quad \leq\left[1-\left(\frac{A_{s 2}^{\beta_{2}}}{\sum_{k=1}^{n} A_{k 2}^{\beta_{2}}}-\frac{A_{s 1}^{\beta_{1}}}{\sum_{k=1}^{n} A_{k 1}^{\beta_{1}}}\right)^{2}\right]^{1 / \beta^{*}} .
\end{aligned}
$$

So, we have the desired inequality (10). The proof of inequalities (11) and (12) is similar to the one of inequality (10), and we omit it. The proof of Theorem 2 is completed.

Putting $s=1$ in (10), (11), and (12), respectively, we obtain the following corollary.

Corollary 3. Let $A_{i j} \geq 0(i=1,2, \ldots, n, j=1,2, \ldots, m)$.

(a) If $\beta_{j}>0(j=1,2, \ldots, m)$ and if $\sum_{j=1}^{m}\left(1 / \beta_{j}\right) \geq 1$, then

$$
\begin{aligned}
\sum_{i=1}^{n} \prod_{j=1}^{m} A_{i j} \leq & {\left[\prod_{j=1}^{m}\left(\sum_{i=1}^{n} A_{i j}^{\beta_{j}}\right)^{1 / \beta_{j}}\right] } \\
& \times\left[1-\left(\frac{A_{12}^{\beta_{2}}}{\sum_{i=1}^{n} A_{i 2}^{\beta_{2}}}-\frac{A_{11}^{\beta_{1}}}{\sum_{i=1}^{n} A_{i 1}^{\beta_{1}}}\right)^{2}\right]^{1 / \beta^{*}},
\end{aligned}
$$

where $\beta^{*}=\max \left\{\beta_{1}, \beta_{2}\right\}$.

(b) If $\beta_{1}>0, \beta_{j}<0(j=2,3, \ldots, m)$ and if $\sum_{j=1}^{m}\left(1 / \beta_{j}\right) \leq$ 1 , then

$$
\begin{aligned}
\sum_{i=1}^{n} \prod_{j=1}^{m} A_{i j} \geq & {\left[\prod_{j=1}^{m}\left(\sum_{i=1}^{n} A_{i j}^{\beta_{j}}\right)^{1 / \beta_{j}}\right] } \\
& \times\left[1-\left(\frac{A_{12}^{\beta_{2}}}{\sum_{i=1}^{n} A_{i 2}^{\beta_{2}}}-\frac{A_{11}^{\beta_{1}}}{\sum_{i=1}^{n} A_{i 1}^{\beta_{1}}}\right)^{2}\right]^{1 / \beta_{2}} .
\end{aligned}
$$


(c) If $\beta_{j}<0(j=1,2, \ldots, m)$, then

$$
\begin{aligned}
\sum_{i=1}^{n} \prod_{j=1}^{m} A_{i j} \geq & {\left[\prod_{j=1}^{m}\left(\sum_{i=1}^{n} A_{i j}^{\beta_{j}}\right)^{1 / \beta_{j}}\right] } \\
& \times\left[1-\left(\frac{A_{12}^{\beta_{2}}}{\sum_{i=1}^{n} A_{i 2}^{\beta_{2}}}-\frac{A_{11}^{\beta_{1}}}{\sum_{i=1}^{n} A_{i 1}^{\beta_{1}}}\right)^{2}\right]^{1 / \beta_{2}} .
\end{aligned}
$$

\section{Application}

In this section, we present a refinement of Beckenbach-type inequality by using Corollary 3. The classical Beckenbach inequality was proved by Beckenbach in [5]. Since Beckenbach discovered this inequality, it has been discussed by many researchers, who either improved it using various techniques or generalized it in many different ways. The interested reader may refer to $[7,16]$ and references therein. In 1983, Wang [15] established the following Beckenbach-type inequality.

Theorem B. Let $f(x)$ and $g(x)$ be positive integrable functions defined on $[0, T]$, and let $(1 / p)+(1 / q)=1$. If $q \geq p>1$, then, for any positive numbers $a, b$, and $c$, the inequality

$$
\frac{\left(a+c \int_{0}^{T} h^{p}(x) d x\right)^{1 / p}}{b+c \int_{0}^{T} h(x) g(x) d x} \leq \frac{\left(a+c \int_{0}^{T} f^{p}(x) d x\right)^{1 / p}}{b+c \int_{0}^{T} f(x) g(x) d x}
$$

holds, where $h(x)=((\operatorname{ag}(x)) / b)^{q / p}$. The sign of the inequality in (21) is reversed if $0<p<1$.

Theorem 4. Let $f(x)$ and $g(x)$ be positive integrable functions defined on $[0, T]$, and let $(1 / p)+(1 / q)=1$. If $q \geq p>1$, then, for any positive numbers $a, b$, and $c$, the inequality

$$
\begin{aligned}
& \frac{\left(a+c \int_{0}^{T} h^{p}(x) d x\right)^{1 / p}}{b+c \int_{0}^{T} h(x) g(x) d x} \\
& \leq \frac{\left(a+c \int_{0}^{T} f^{p}(x) d x\right)^{1 / p}}{b+c \int_{0}^{T} f(x) g(x) d x} \\
& \quad \times\left[1-\left(\frac{a^{-(q / p)} b^{q}}{a^{-(q / p)} b^{q}+c \int_{0}^{T} g^{q}(x) d x}\right.\right. \\
& \left.\left.-\frac{a}{a+c \int_{0}^{T} f^{p}(x) d x}\right)^{2}\right]^{1 / q}
\end{aligned}
$$

holds, where $h(x)=((\operatorname{ag}(x)) / b)^{q / p}$. The sign of the inequality in (22) is reversed if $0<p<1$.
Proof. After some simple calculations, we have

$$
\frac{\left(a+c \int_{0}^{T} h^{p}(x) d x\right)^{1 / p}}{b+c \int_{0}^{T} h(x) g(x) d x}=\left(a^{-(q / p)} b^{q}+c \int_{0}^{T} g^{q}(x) d x\right)^{-1 / q}
$$

On the other hand, putting $\beta_{1}=p, \beta_{2}=q, m=2$ in (18), from the integral form of Hölder's inequality (1) and Corollary 3 , we obtain

$$
\begin{aligned}
b+c & \int_{0}^{T} f(x) g(x) d x \\
\leq & b+c\left(\int_{0}^{T} f^{p}(x) d x\right)^{1 / p}\left(\int_{0}^{T} g^{q}(x) d x\right)^{1 / q} \\
= & a^{1 / p}\left(b a^{-1 / p}\right)+\left(c \int_{0}^{T} f^{p}(x) d x\right)^{1 / p} \\
& \times\left(c \int_{0}^{T} g^{q}(x) d x\right)^{1 / q} \\
\leq & \left(a+c \int_{0}^{T} f^{p}(x) d x\right)^{1 / p} \\
& \times\left(a^{-(q / p)} b^{q}+c \int_{0}^{T} g^{q}(x) d x\right)^{1 / q} \\
& \times\left[1-\left(\frac{-a^{-(q / p)} b^{q}}{a+c \int_{0}^{T} f^{p}(x) d x}\right)^{2}\right]^{1 / q} \\
& {\left[\quad b^{q}+c \int_{0}^{T} g^{q}(x) d x\right.}
\end{aligned}
$$

that is,

$$
\begin{gathered}
\left(a^{-(q / p)} b^{q}+c \int_{0}^{T} g^{q}(x) d x\right)^{-1 / q} \\
\leq \frac{\left(a+c \int_{0}^{T} f^{p}(x) d x\right)^{1 / p}}{b+c \int_{0}^{T} f(x) g(x) d x} \\
\times\left[1-\left(\frac{a^{-(q / p)} b^{q}}{a^{-(q / p)} b^{q}+c \int_{0}^{T} g^{q}(x) d x}\right.\right. \\
\left.\left.-\frac{a}{a+c \int_{0}^{T} f^{p}(x) d x}\right)^{2}\right]^{1 / q} .
\end{gathered}
$$

Combining inequalities (23) and (25) yields inequality (22). In a similar way, we can prove that the reversed version of inequality (22) is true. Thus, the proof of Theorem 4 is complete. 


\section{Acknowledgments}

The author would like to sincerely declare his special thanks to both the anonymous referees for their helpful comments and suggestions. This work was supported by the NNSF of China (Grant no. 61073121) and the Fundamental Research Funds for the Central Universities (Grant no. 13ZD19).

\section{References}

[1] S. Barza, D. Kravvaritis, and N. Popa, "Matriceal Lebesgue spaces and Hölder inequality," Journal of Function Spaces and Applications, vol. 3, no. 3, pp. 239-249, 2005.

[2] L. Nikolova and S. Varošanec, "Refinements of Hölder's inequality derived from functions $\psi_{p, q, \lambda}$ and $\varphi_{p, q, \lambda}$," Annals of Functional Analysis, vol. 2, no. 1, pp. 72-83, 2011.

[3] J. Tian and X.-M. Hu, "A new reversed version of a generalized sharp Hölder's inequality and its applications," Abstract and Applied Analysis, vol. 2013, Article ID 901824, 9 pages, 2013.

[4] S. Abramovich, J. Pečarić, and S. Varošanec, "Continuous sharpening of Hölder's and Minkowski's inequalities," Mathematical Inequalities \& Applications, vol. 8, no. 2, pp. 179-190, 2005.

[5] E. F. Beckenbach, "On Hölder's inequality," Journal of Mathematical Analysis and Applications, vol. 15, pp. 21-29, 1966.

[6] H. Ibrahim, "A generalization of a logarithmic Sobolev inequality to the Hölder class," Journal of Function Spaces and Applications, vol. 2012, Article ID 148706, 15 pages, 2012.

[7] D. S. Mitrinović, J. E. Pečarić, and A. M. Fink, Classical and New Inequalities in Analysis, vol. 61 of Mathematics and its Applications, Kluwer Academic Publishers, Dordrecht, The Netherlands, 1993.

[8] J. Tian, "Extension of Hu Ke's inequality and its applications," Journal of Inequalities and Applications, vol. 2011, article 77, 2011.

[9] J. Tian, "Reversed version of a generalized sharp Hölder's inequality and its applications," Information Sciences, vol. 201, pp. 61-69, 2012.

[10] J. Tian, "Reversed version of a generalized Aczel's inequality and its application," Journal of Inequalities and Applications, vol. 2012, article 202, 2012.

[11] J. Tian, "Property of a Holder-type inequality and its application," Mathematical Inequalities and Applications, vol. 16, no. 3, pp. 831-841, 2013.

[12] J. Tian and X. M. Hu, "Renements of generalized Hölder's inequality," Journal of Mathematical Inequalities. In press.

[13] J. Tian and S. Wang, "Renements of generalized Aczel's inequality and Bellman's inequality and their applications," Journal of Applied Mathematics, vol. 2013, Article ID 645263, 6 pages, 2013.

[14] P. M. Vasić and J. E. Pečarić, "On the Jensen inequality for monotone functions," Analele Universităţii din Timişoara, Seria Ştiinţe Matematice, vol. 17, no. 1, pp. 95-104, 1979.

[15] C.-L. Wang, "Characteristics of nonlinear positive functionals and their applications," Journal of Mathematical Analysis and Applications, vol. 95, no. 2, pp. 564-574, 1983.

[16] G. H. Hardy, J. E. Littlewood, and G. Pólya, Inequalities, Cambridge University Press, Cambridge, UK, 2nd edition, 1952. 


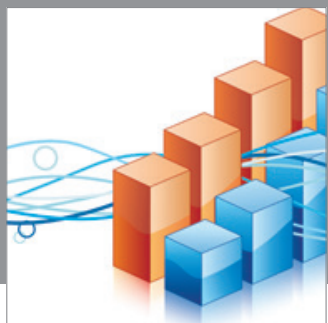

Advances in

Operations Research

mansans

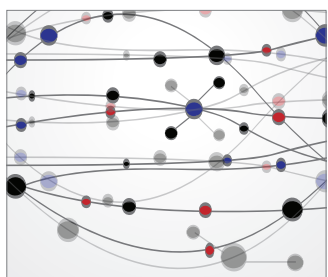

The Scientific World Journal
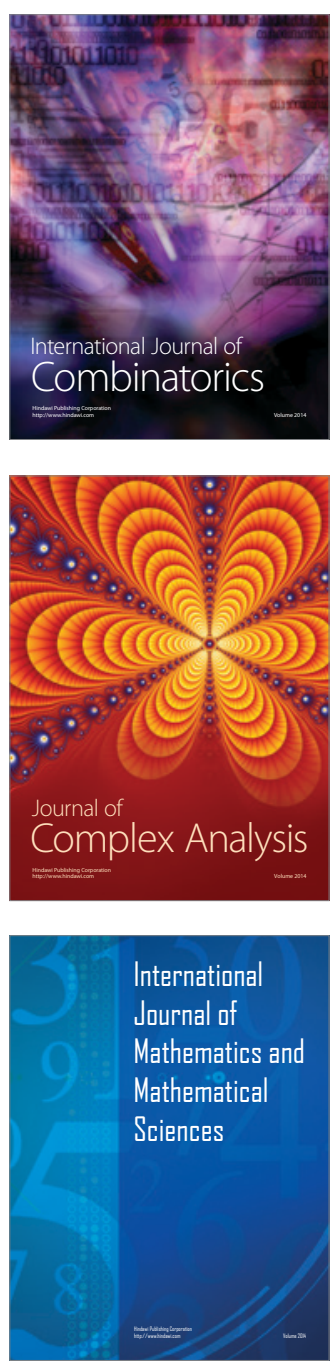
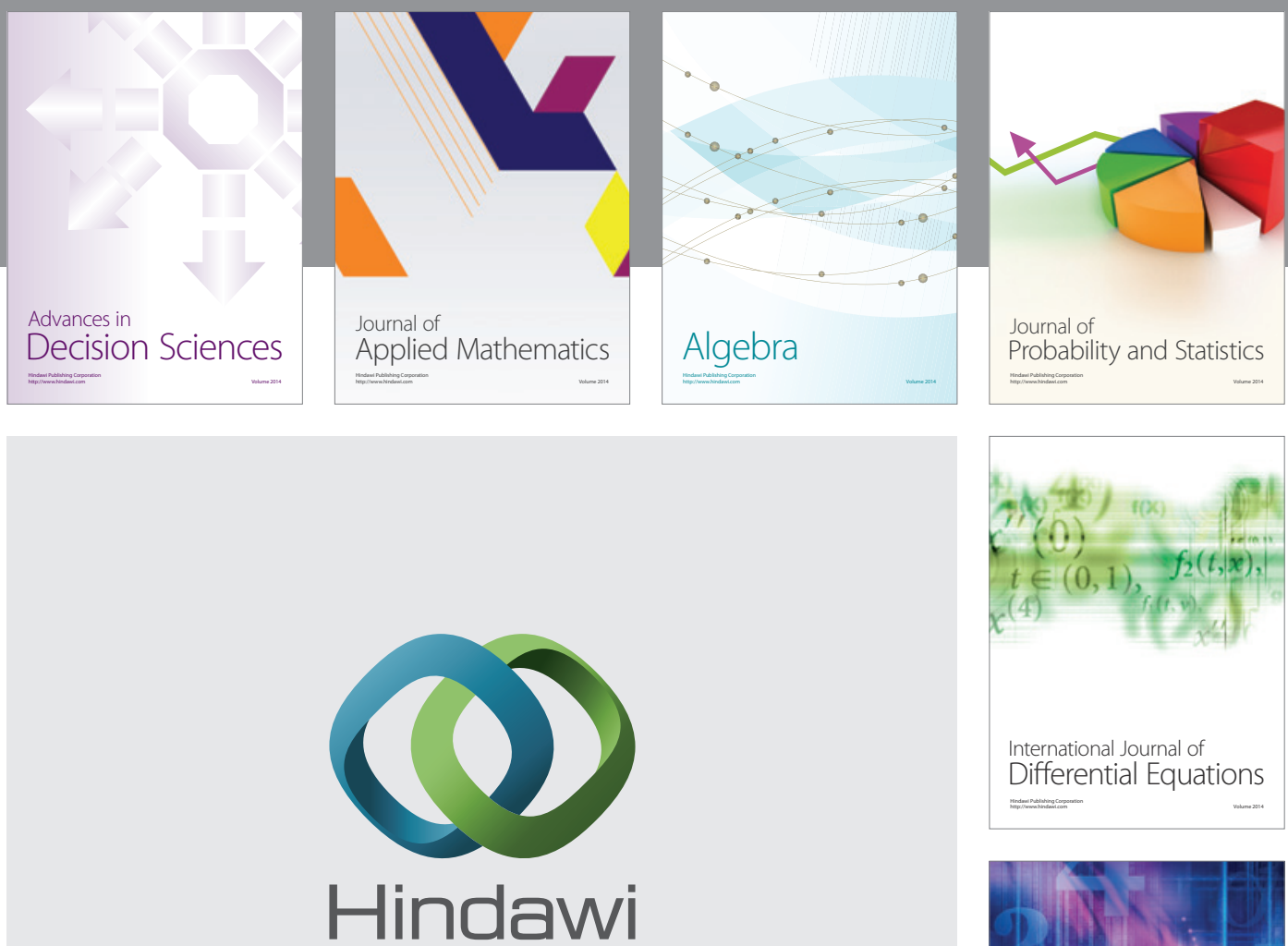

Submit your manuscripts at http://www.hindawi.com
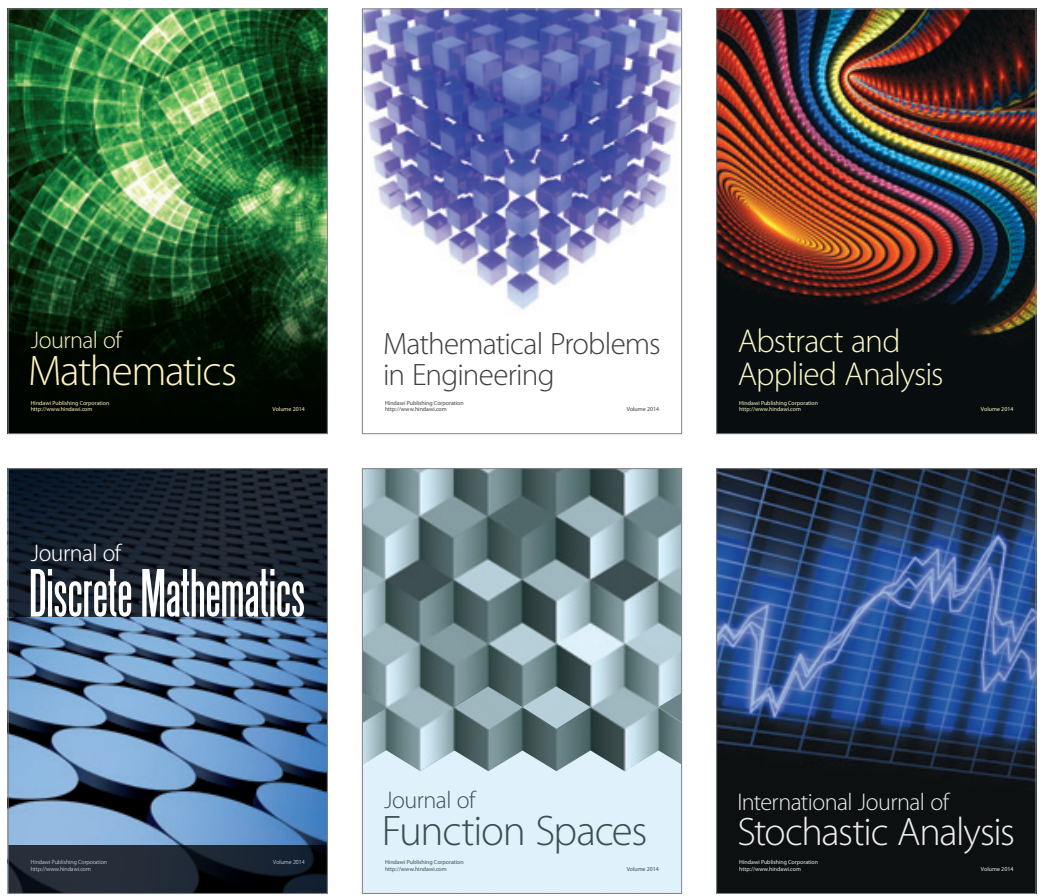

Journal of

Function Spaces

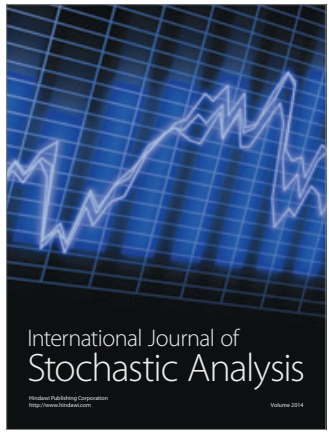

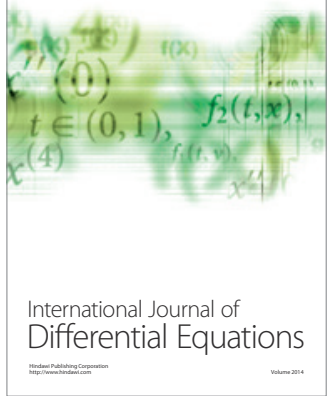
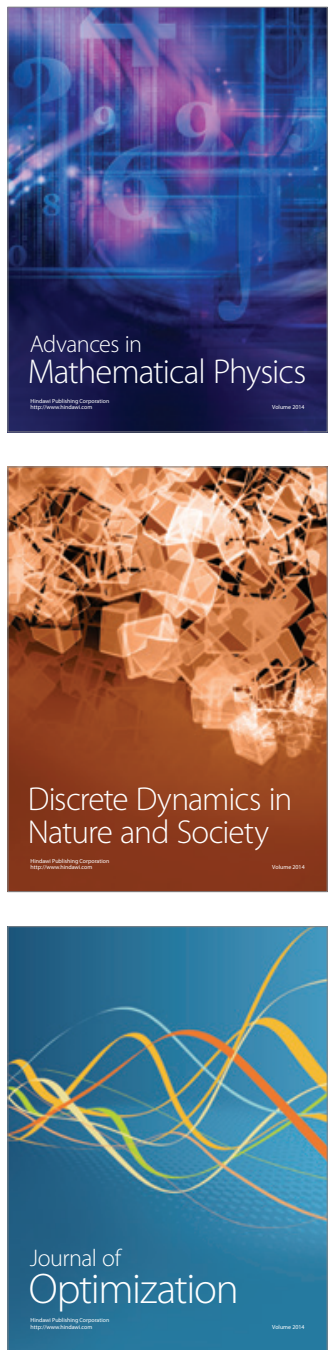